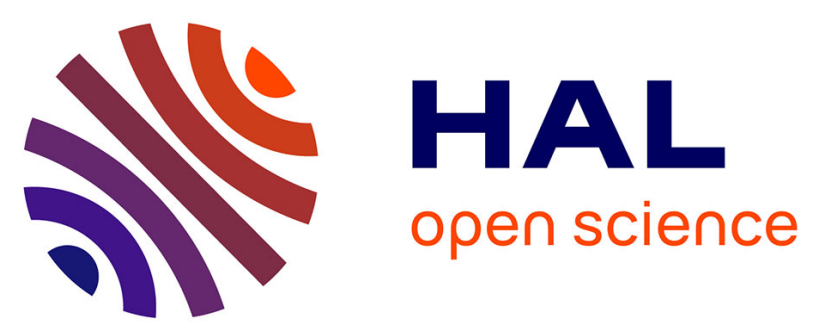

\title{
ON THE AUTOMATICITY OF SEQUENCES DEFINED BY THE THUE-MORSE AND PERIOD-DOUBLING STIELTJES CONTINUED FRACTIONS
}

Yining Hu, Guoniu Wei-Han

\section{To cite this version:}

Yining Hu, Guoniu Wei-Han. ON THE AUTOMATICITY OF SEQUENCES DEFINED BY THE THUE-MORSE AND PERIOD-DOUBLING STIELTJES CONTINUED FRACTIONS. International Journal of Number Theory, 2020, pp.1-26. 10.1142/S1793042120501134 . hal-02968071

\section{HAL Id: hal-02968071 \\ https://hal.science/hal-02968071}

Submitted on 15 Oct 2020

HAL is a multi-disciplinary open access archive for the deposit and dissemination of scientific research documents, whether they are published or not. The documents may come from teaching and research institutions in France or abroad, or from public or private research centers.
L'archive ouverte pluridisciplinaire HAL, est destinée au dépôt et à la diffusion de documents scientifiques de niveau recherche, publiés ou non, émanant des établissements d'enseignement et de recherche français ou étrangers, des laboratoires publics ou privés. 


\title{
ON THE AUTOMATICITY OF SEQUENCES DEFINED BY THE THUE-MORSE AND PERIOD-DOUBLING STIELTJES CONTINUED FRACTIONS
}

\author{
YINING HU AND GUONIU WEI-HAN
}

\begin{abstract}
Continued fraction expansions of automatic numbers have been extensively studied during the last few decades. The research interests are, on one hand, in the degree or automaticity of the partial quotients following the seminal paper of Baum and Sweet in 1976, and on the other hand, in calculating the Hankel determinants and irrationality exponents, as one can find in the works of Allouche-Peyrière-Wen-Wen, Bugeaud, and the first author. The present paper is motivated by the converse problem: to study Stieltjes continued fractions whose coefficients form an automatic sequence. We consider two such continued fractions defined by the Thue-Morse and period-doubling sequences, respectively, and prove that they are congruent to algebraic series in $\mathbb{Z}[[x]]$ modulo 4 . Consequently, the sequences of the coefficients of the power series expansions of the two continued fractions modulo 4 are 2-automatic.
\end{abstract}

\section{INTRODUCTION}

Continued fraction expansions of automatic numbers have been extensively studied during the last few decades. By the well-known theorem of Christol [14], an element in $\mathbb{F}_{q}((1 / x))$ is algebraic over $\mathbb{F}_{q}(x)$ if and only if its coefficients form a $q$-automatic sequence. In 1976, Baum and Sweet [7] proved that the continued fraction expansion of the unique solution $\varphi(x)$ in $\mathbb{F}_{2}((1 / x))$ of the equation

$$
x f^{3}+f+x=0,
$$

has partial quotients of bounded degree. They also gave examples of algebraic elements of degree greater than 2 in $\mathbb{F}_{q}((1 / x))$ whose continued fraction expansion have partial quotients of unbounded degree. In contrast, we do not know any algebraic real number of degree greater than 2 that has bounded or unbounded partial quotients. For every even degree $d$, Baum and Sweet [8] gave examples of elements in $\mathbb{F}_{2}((1 / x))$, algebraic of degree $d$, with bounded partial quotients. Mills and Robbins [29] explicitly gave the continued fraction expansion of $\varphi(x)$. They also gave examples, for each odd prime $p$, of algebraic elements of degree greater than 2 in $\mathbb{F}_{p}((1 / x))$ whose partial quotients are linear; this was largely generalized by Lasjaunias and Yao [26]. Allouche proved [1] that the sequences of partial quotients for the examples in the article of Mills and Robbins [29] are automatic. In contrast, Mkaouar [?] proved that the sequence of partial quotients of $\varphi(x)$, while being morphic, is not automatic. Lasjaunias and Yao [27, 28] considered

Date: August 10, 2020.

2010 Mathematics Subject Classification. 11B85, 11J70, 11B50, 11Y65, 05A15.

Key words and phrases. automatic sequence, continued fraction, Hankel determinant, ThueMorse sequence, period-doubling sequence. 
the sequence of leading coefficients of the partial quotients instead of the partial quotients themselves and described several families of hyperquadratic series for which the leading coefficients of the partial quotients form automatic sequences.

On the other hand, continued fraction expansions of automatic numbers have been studied for calculating Hankel determinants and irrationality exponents [22, 23, 13, 19, 6]. In 1998, Allouche, Peyrière, Wen and Wen proved that all the Hankel determinants of the Thue-Morse sequence are nonzero [3]. This property allowed Bugeaud to prove that the irrationality exponents of the Thue-Morse-Mahler numbers are exactly 2 [10]. Since then, the Hankel determinants for several other automatic sequences, in particular, the paperfolding sequence, the Stern sequence and the period-doubling sequence, were studied by Coons, Vrbik, Guo, Wu, Wen, Bugeaud and $\mathrm{Fu}[15,21,20,12]$. Using Jacobi continued fractions, the first author found a simple proof of APWW's result [22]. Finally, the Euler-Lagrange theorem says that the continued fraction expansion of a quadratic irrational number is ultimately periodic. The first author obtained a similar result for quadratic power series on finite fields and proved that their Hankel determinants are ultimately periodic [23].

The present paper is motivated by the converse problem: to study Stieltjes continued fractions whose coefficients form an automatic sequence.

We now give a brief introduction to automatic sequences. We refer the readers to the book of Allouche and Shallit [5, p. 185] for a comprehensive exposition. Automatic sequences appear naturally in the study of various domains of mathematics and theoretical computer science. One of the equivalent definitions of automatic sequences is the following: for an integer $k \geq 2$, a sequence $\left(u_{n}\right)_{n \geq 0}$ is said to be $k$-automatic if its $k$-kernel, defined as

$$
\left\{\left(u\left(k^{d} n+j\right)\right)_{n \geq 0} \mid d \in \mathbb{N}, 0 \leq j \leq k^{d}-1\right\},
$$

is finite. Thus, if we let $\Lambda_{j}$ denote the Cartier operators [5, p. 376] that maps $\sum_{n=0}^{\infty} a_{n} x^{n}$ to $\sum_{n=0}^{\infty} a_{k n+j} x^{n}$, then the $k$-kernel of $\left(u_{n}\right)_{n \geq 0}$ is in bijection with the smallest set containing the series $\sum_{n=0}^{\infty} u_{n} x^{n}$ that is stable under the operations of $\Lambda_{j}(j=0,1, \ldots, k-1)$. We use a double list $L$ to encode the structure of the $k$-kernel. Suppose that the $k$-kernel of a sequence is the set $\left\{s_{0}, s_{1}, \ldots, s_{n}\right\}$ with $s_{0}$ being the sequence itself. By $L[i][j]=l$ we mean that $s_{i}$ is mapped to $s_{l}$ by $\Lambda_{j}$. This double list gives the transition function of a $k$-automaton (reading from the least significant digit) of the sequence.

In this article we will consider the Thue-Morse sequence $\mathbf{t}=\left(t_{n}\right)$ defined by the recurrence relations (see $[33,4]$ )

$$
\begin{aligned}
t_{0} & =1 ; & & \\
t_{2 n} & =t_{n} ; & & (n \geq 1) \\
t_{2 n+1} & =-t_{n}, & & (n \geq 0)
\end{aligned}
$$

and the period-doubling sequence $\mathbf{s}=\left(s_{n}\right)$ defined by the recurrence relations $[31,19]$

$$
\begin{aligned}
s_{2 n} & =1 ; & & (n \geq 0) \\
s_{2 n+1} & =-s_{n} . & & (n \geq 0)
\end{aligned}
$$

We see from the definition that the 2-kernel of the Thue-Morse sequence is

$$
\left\{\left(t_{n}\right)_{n},\left(t_{2 n+1}\right)_{n}\right\},
$$


and the 2-kernel of the period-doubling sequence is

$$
\left\{\left(s_{n}\right)_{n},\left(s_{2 n}\right)_{n},\left(s_{2 n+1}\right)_{n},\left(s_{4 n+1}\right)_{n}\right\} .
$$

Therefore they are both 2-automatic. The structures of the above two 2-kernels are represented by $[[0,1],[1,0]]$ and $[[1,2],[1,1],[3,0],[3,3]]$ respectively.

We consider two cases of formal power series defined by automatic Stieltjes continued fractions and compare our result to what is known for real numbers defined by automatic continued fractions. Basic definitions and properties of Stieltjes and Jacobi continued fractions will be recalled in Section 2. We define the Stieltjes continued fractions by the Thue-Morse and the period-doubling sequence:

$$
C(x):=\sum_{n \geq 0} c_{n} x^{n}:=\frac{t_{0}}{1+\frac{t_{1} x}{1+\frac{t_{2} x}{1+\frac{t_{3} x}{1+\frac{t_{4} x}{\ddots}}}}}=\frac{1}{1-\frac{x}{1-\frac{x}{1+\frac{x}{1-\frac{x}{\ddots}}}}}
$$

and

$$
D(x):=\sum_{n \geq 0} d_{n} x^{n}:=\frac{s_{0}}{1+\frac{s_{1} x}{1+\frac{s_{2} x}{1+\frac{s_{3} x}{1+\frac{s_{4} x}{\ddots}}}}}=\frac{1}{1-\frac{x}{1+\frac{x}{1+\frac{x}{1+\frac{x}{\ddots}}}}} .
$$

The above two continued fractions will be called Thue-Morse continued fraction and Period-doubling continued fraction respectively. Write $\bar{c}_{n}=\pi\left(c_{n}\right), \bar{C}(x)=$ $\sum_{n \geq 0} \bar{c}_{n} x^{n}$, and $\bar{d}_{n}=\pi\left(d_{n}\right), \bar{D}(x)=\sum_{n \geq 0} \bar{d}_{n} x^{n}$, where $\pi$ is the canonical surjection of $\mathbb{Z}$ onto $\mathbb{Z} / 4 \mathbb{Z}$. Note that we could also have defined $\bar{C}(x)$ and $\bar{D}(x)$ by (1.1) and (1.2) while viewing \pm 1 as elements in $\mathbb{Z} / 4 \mathbb{Z}$. The first terms of these sequences are listed below.

$$
\begin{aligned}
& \left(t_{n}\right)=(1,-1,-1,1,-1,1,1,-1,-1,1,1,-1,1,-1,-1,1, \ldots), \\
& \left(c_{n}\right)=(1,1,2,3,4,6,8,11,14,18,20,22,16,4,-32,-93,-220, \ldots), \\
& \left(\bar{c}_{n}\right)=(1,1,2,3,0,2,0,3,2,2,0,2,0,0,0,3,0,2,0,2,0,0,0, \ldots), \\
& \left(s_{n}\right)=(1,-1,1,1,1,-1,1,-1,1,-1,1,1,1,-1,1,1, \ldots), \\
& \left(d_{n}\right)=(1,1,0,1,-2,4,-8,17,-36,74,-152,316,-656,1352, \ldots), \\
& \left(\bar{d}_{n}\right)=(1,1,0,1,2,0,0,1,0,2,0,0,0,0,0,1,2,0,0,2,0,0,0,0, \ldots) .
\end{aligned}
$$

In the present paper we study the above two continued fractions and obtain the following properties of the sequences $\left(\bar{c}_{n}\right)$ and $\left(\bar{d}_{n}\right)$.

Theorem 1.1. We have the following congruence:

$$
C(x) \equiv \frac{\sqrt{1-4 x}-1}{2 x}+1+\sqrt{2 \sqrt{1-4 x}-1}(\bmod 4) .
$$


Theorem 1.2. We have the following congruence:

$$
D(x) \equiv \frac{(1+\sqrt{1+4 x}) \sqrt{2 \sqrt{1-4 x^{2}}-1}-2}{2 x}(\bmod 4) .
$$

The following theorem [16] then allows us to conclude that $\left(\bar{c}_{n}\right)_{n}$ and $\left(\bar{d}_{n}\right)_{n}$ are 2-automatic.

Theorem 1.3 (Denef-Lipschitz). Suppose that the power series $f\left(x_{1}, \ldots, x_{k}\right) \in$ $\mathbb{Z}_{p}\left[\left[x_{1}, \ldots, x_{k}\right]\right]$ is algebraic over $\mathbb{Z}_{p}\left[x_{1}, \ldots, x_{k}\right]$. Then for each $\alpha$, the coefficient sequence of $f\left(\bmod p^{\alpha}\right)$ is p-automatic.

The automaticity of $\left(\bar{c}_{n}\right)_{n}$ and $\left(\bar{d}_{n}\right)_{n}$ can also be proved by a direct calculation of their 2-kernels.

Theorem 1.4. The sequence $\left(\bar{c}_{n}\right)$ is 2-automatic; the structure of its 2-kernel is represented by $[[1,2],[3,4],[5,6],[1,7],[4,7],[5,4],[8,6],[7,7],[8,4]]$.

Theorem 1.5. The sequence $\left(\bar{d}_{n}\right)$ is 2-automatic; the structure of its 2-kernel is represented by $[[1,0],[2,3],[1,4],[3,3],[4,3]]$

The right hand side of congruence (1.3) and (1.4) are respectively of degree 4 and 8 over $\mathbb{Z}(x)$. This raises the question of what the minimal degree of polynomial equations that $\bar{C}$ and $\bar{D}$ satisfy is. Concerning this, we have the following result.

Theorem 1.6. Let $S(x, y)=\left(x y^{2}+y+1\right)^{2} \in \mathbb{Z} / 4 \mathbb{Z}[x, y]$. Then for both series $\bar{C}(x)$ and $\bar{D}(x)$ in $\mathbb{Z} / 4 \mathbb{Z}$, we have $S(x, \bar{C}(x))=S(x, \bar{D}(x))=0$. Furthermore, there is no polynomial in $\mathbb{Z} / 4 \mathbb{Z}[x, y]$ that, seen as a polynomial in $y$, has degree less than 4 , and, whose leading coefficient is invertible in the ring of Laurent series $\mathbb{Z} / 4 \mathbb{Z}((x))$, that annihilates either $\bar{C}(x)$ or $\bar{D}(x)$.

Informally put, Theorem 1.6 says that $\bar{C}(x)$ and $\bar{D}(x)$ are of degree 4 , while their continued fraction expansion are 2-automatic. It may be interesting to compare Theorem 1.6 to the following result concerning automatic sequences and real continued fractions [11].

Theorem 1.7 (Bugeaud 2013). The continued fraction expansion of an algebraic number of degree at least three cannot be generated by a finite automaton.

The Hankel determinant of order $n$ of the formal power series $f(x)=a_{0}+a_{1} x+$ $a_{2} x^{2}+\cdots$ (or of the sequence $\left(a_{0}, a_{1}, a_{2}, \cdots\right)$ is defined by

$$
H_{n}(f(x))=H_{n}\left(a_{0}, a_{1}, a_{2}, \ldots\right):=\operatorname{det}\left(a_{i+j}\right)_{0 \leq i, j \leq n-1}
$$

for $n \geq 1$, and $H_{0}(f(x))=H_{0}\left(a_{0}, a_{1}, a_{2}, \ldots\right)=1$ if $n=0$.

Concerning the Hankel determinants of $C(x)$ and $D(x)$, we have the following result.

Theorem 1.8. The sequences of Hankel determinants $\left(H_{n}(C(x))\right)$ and $\left(H_{n}(D(x))\right)$ are 2-automatic.

Based on our results, we put forward the following conjecture.

Conjecture 1.9. The sequences $c_{n}\left(\bmod 2^{m}\right)$ and $d_{n}\left(\bmod 2^{m}\right)$ are 2-automatic for all $m \geq 1$. 
Theorem 1.4 and 1.5 says that Conjecture 1.9 is true for $m=2$. Note that if the conjecture is true for $m=k$, then it is also true for all positive integers $m<k$. For $m=1$, we can also see directly that

$$
C(x) \equiv D(x) \equiv \frac{1}{1-\frac{x}{1-\frac{x}{1-\frac{x}{\ddots}}}}(\bmod 2) .
$$

The right hand side of the congruence is the generating function for the Catalan numbers [9]. Being quadratic, it is 2-automatic modulo 2.

When $m=3$, we have the following conjecture.

Conjecture 1.10. The sequences $c_{n}\left(\bmod 2^{3}\right)$ and $d_{n}\left(\bmod 2^{3}\right)$ are 2-automatic with the following kernel structure for $c_{n}\left(\bmod 2^{3}\right)$

$$
\begin{gathered}
{[[1,2],[3,4],[5,6],[7,8],[9,10],[11,12],[13,6],[3,14],[8,10],[4,8],} \\
[10,10],[11,15],[12,8],[16,12],[17,10],[15,8],[16,15],[14,8]] ;
\end{gathered}
$$

and for $d_{n}\left(\bmod 2^{3}\right)$

$$
\begin{gathered}
{[[1,2],[3,4],[5,2],[6,7],[4,4],[8,9],[3,9],[10,4],[11,12],} \\
[9,4],[7,9],[8,4],[13,4],[12,9]] .
\end{gathered}
$$

This article is structured as follows: in Section 2, we give the definitions and properties of Stieltjes and Jacobi continued fractions. In Section 3, we exploit the structure of the Thue-Morse sequence and obtain the relations between certain subsequences of $P_{n}(x)$ and $Q_{n}(x)$, with $P_{n}(x) / Q_{n}(x)$ being the canonical representation of the $n$-th convergent of the continued fraction $C(x)$. Then we prove by induction the explicit expression of eight subsequences. We only use two of them but we need all eight for the induction hypotheses. Taking the limit, we obtain the explicit expression of the Thue-Morse continued fraction $\bar{C}(x)$ as a power series and prove that it is equal to an algebraic series with integer coefficients modulo 4 . In consequence, its coefficients form a 2-automatic sequence. In Section 4 we obtain similar results for the period-doubling continued fraction $\bar{D}(x)$ using what we have proved for $\bar{C}(x)$ and the relation between the Thue-Morse and the period-doubling sequences. In Section 5 we prove Theorem 1.6. Finally in Section 6 we prove that the sequences of Hankel determinants $H_{n}(C(x))$ and $H_{n}(D(x))$ are 2-automatic.

\section{Stieltues and Jacobi Continued fractions}

Stieltjes and Jacobi continued fractions are studied in enumerative combinatorics for their link with orthogonal polynomials and weighted Motzkin paths (see [25, p. 386 , p. 389], [34], [17]). For a sequence $\mathbf{a}=\left(a_{n}\right)_{n}$ taking values in a field $\mathbb{K}$, and 
for each positive integer $n$, we define the rational fraction:

$$
\operatorname{Stiel}_{n}(\mathbf{a}):=\frac{a_{0}}{1+\frac{a_{1} x}{1+\frac{a_{2} x}{\frac{\ddots}{1+\frac{a_{n-1} x}{1+a_{n} x}}}}}
$$

which we also write as $\llbracket a_{0}, a_{1}, \ldots, a_{n} \rrbracket$ for short.

We define two sequence of polynomials $P_{n}(x)$ and $Q_{n}(x)$ by the initial conditions $P_{0}(x)=a_{0}, Q_{0}(x)=1, P_{1}(x)=a_{0}$ and $Q_{1}(x)=1+a_{1} x$, and for $n \geq 2$

$$
\left(\begin{array}{cc}
1 & a_{n} x \\
1 & 0
\end{array}\right) \cdots\left(\begin{array}{cc}
1 & a_{2} x \\
1 & 0
\end{array}\right)\left(\begin{array}{cc}
P_{1}(x) & Q_{1}(x) \\
P_{0}(x) & Q_{0}(x)
\end{array}\right)=\left(\begin{array}{cc}
P_{n}(x) & Q_{n}(x) \\
P_{n-1}(x) & Q_{n-1}(x)
\end{array}\right) .
$$

We have $\operatorname{Stiel}_{n}(\mathbf{a})=P_{n}(x) / Q_{n}(x)$ for all $n$. A proof of the following theorem can be found in the lecture notes by Foata and the first author [18, p. 257].

Theorem 2.1. The sequence of formal power series $P_{n}(x) / Q_{n}(x)$ is convergent.

The infinite Stieltjes continued fraction $\operatorname{Stiel}(\mathbf{a})$ is defined to be

$$
\lim _{n \rightarrow \infty} P_{n}(x) / Q_{n}(x),
$$

the rational fraction $P_{n}(x) / Q_{n}(x)$ is called the $n$-th convergent of $\operatorname{Stiel}(\mathbf{a})$ and the unsimplified fraction $P_{n}(x) / Q_{n}(x)$ the canonical representation of $\operatorname{Stiel}_{n}(\mathbf{a})$.

For $0 \leq k<n$, if $P(x) / Q(x)$ is the canonical representation of the Stieltjes continued fraction $\llbracket a_{k}, \ldots, a_{n} \rrbracket$, then it can be easily shown from $(2.2)$ that

$$
\begin{aligned}
P_{n}(x) & =Q(x) P_{k-1}(x)+x P(x) P_{k-2}(x), \\
Q_{n}(x) & =Q(x) Q_{k-1}(x)+x P(x) Q_{k-2}(x) .
\end{aligned}
$$

We define the Jacobi continued fractions in a similar way. For two sequences $\mathbf{u}=\left(u_{n}\right)_{n}$ and $\mathbf{v}=\left(v_{n}\right)_{n}$ with $v_{i} \neq 0$ for all $i \in \mathbb{N}, \operatorname{Jac}(\mathbf{u}, \mathbf{v})$ is defined to be the infinite continued fraction

$$
\operatorname{Jac}(\mathbf{u}, \mathbf{v})=\frac{v_{0}}{1+u_{1} x-\frac{v_{1} x^{2}}{1+u_{2} x-\frac{v_{2} x^{2}}{1+u_{3} x-\frac{v_{3} x^{2}}{\ddots}}}}
$$

The basic properties on Stieltjes and Jacobi continued fractions can be found in the works of Flajolet, Wall, Stieltjes and Heilermann [17, 35, 32, 24]. We emphasize the fact that the Hankel determinants can be calculated from Stieltjes and Jacobi continued fractions by means of the following fundamental relation, first stated by Heilermann in 1846 [24]:

Theorem 2.2. The nth-order Hankel determinants of the Stieltjes (2.1) and Jacobi (2.5) continued fractions are given by

$$
\begin{aligned}
H_{n}(\operatorname{Stiel}(\mathbf{a})) & =a_{0}^{n}\left(a_{1} a_{2}\right)^{n-1}\left(a_{3} a_{4}\right)^{n-2} \cdots\left(a_{2 n-3} a_{2 n-2}\right), \\
H_{n}(\operatorname{Jac}(\mathbf{u}, \mathbf{v})) & =v_{0}^{n} v_{1}^{n-1} v_{2}^{n-2} \cdots v_{n-2}^{2} v_{n-1} .
\end{aligned}
$$


The following contraction theorem establishes a link between Stieltjes and Jacobi continued fractions [35, 30,32].

Theorem 2.3. [Contraction Theorem] The Stieltjes continued fraction Stiel(a) and Jacobi continued fraction $\operatorname{Jac}(\mathbf{u}, \mathbf{v})$ are equal, if

$$
\begin{array}{lll}
u_{1} & =a_{1} ; & \\
u_{k} & =a_{2 k-2}+a_{2 k-1} ; & \\
v_{0}=a_{0} ; & (k \geq 2) \\
v_{k}=a_{2 k-1} a_{2 k} . & (k \geq 1)
\end{array}
$$

Using the above notation, the two power series $C(x)$ and $D(x)$ defined in Section 1 can be written as $C(x)=\operatorname{Stiel}(\mathbf{t})$ and $D(x)=\operatorname{Stiel}(\mathbf{s})$.

\section{Thue-Morse CONTINUED FRACTION}

First we consider the $n$-th convergent $P_{n}(x) / Q_{n}(x)$ of the Thue-Morse continued fraction $C(x)$. Making use of the structure of the Thue-Morse sequence, we establish the following recurrence relations of $P_{n}$ and $Q_{n}$.

Lemma 3.1. Let $P_{n}(x) / Q_{n}(x)$ be the canonical representation of $\operatorname{Stiel}_{n}(\mathbf{t})$. The two sequences $P_{n}(x)$ and $Q_{n}(x)$ are characterized by the initial conditions

$$
P_{0}(x)=P_{1}(x)=Q_{0}(x)=1, Q_{1}(x)=1-x
$$

and the following recurrence relations for $m \geq 1$ and $1 \leq \epsilon \leq 2^{m}$ :

$$
U_{2^{m+1}-\epsilon}(x)=Q_{2^{m}-\epsilon}(-x) U_{2^{m}-1}(x)-x P_{2^{m}-\epsilon}(-x) U_{2^{m}-2}(x),
$$

where $U$ is either of the sequences $P$ or $Q$.

Proof. For a fixed $1 \leq \epsilon \leq 2^{m}$, let $P(x) / Q(x)$ be the canonical representation of the Stieltjes continued fraction $\llbracket t_{2^{m}}, t_{2^{m}+1}, \ldots, t_{2^{m+1}-\epsilon} \rrbracket$. From the definition of the Thue-Morse sequence, we see that $t_{n}=1$ if the number of 1 's in the binary expansion of $n$ is even, and $t_{n}=-1$ otherwise, and therefore $t_{2^{m}+j}=-t_{j}$ for all $m \geq 0$ and $0 \leq j \leq 2^{m}-1$. Hence $P(x) / Q(x)$ is in fact the canonical representation of $\llbracket-t_{0},-t_{1}, \ldots,-t_{2^{m}-\epsilon} \rrbracket$. By Eq. (2.2), $P(x)=-P_{2^{m}-\epsilon}(-x)$ and $Q(x)=Q_{2^{m}-\epsilon}(-x)$. Using formulae (2.3) and (2.4) we get the desired result.

From the above recurrence relations of $P_{n}(x)$ and $Q_{n}(x)$, we are able to derive by induction the explicit expression of $P_{2^{2 m}-2}(x)$ and $Q_{2^{2 m}-2}(x)$, which we will then use to calculate $C(x)=\lim P_{n}(x) / Q_{n}(x)$.

To simplify notation, we define, for $m \geq 0$,

$$
S_{m}(x)=\sum_{j=0}^{m-1} x^{2^{j}}, \quad S_{m}^{e}(x)=\sum_{j=0}^{m-1} x^{2^{2 j}}, \quad S_{m}^{o}(x)=\sum_{j=0}^{m-1} x^{2^{2 j+1}},
$$

and

$$
S_{\infty}(x)=\sum_{j=0}^{\infty} x^{2^{j}}, \quad S_{\infty}^{e}(x)=\sum_{j=0}^{\infty} x^{2^{2 j}}, \quad S_{\infty}^{o}(x)=\sum_{j=0}^{\infty} x^{2^{2 j+1}} .
$$

It is useful to keep in mind that

$$
S_{\infty}(x)^{2} \equiv S_{\infty}\left(x^{2}\right) \equiv x+S_{\infty}(x) \quad(\bmod 2) .
$$

If the parameter is $x$, we write without the parameter as $S_{m}:=S_{m}(x)$, etc. Recall that the Kronecker delta symbol $\delta_{i, j}$ is 1 if $i=j$, and 0 otherwise. 
We are only interested in 3) and 7) from the following proposition, but we need the others for the proof by induction.

Proposition 3.2. We have the following explicit values for the polynomials $P_{n}(x)$ and $Q_{n}(x)$ for $n=2^{k}-1$ and $n=2^{k}-2$.

1) $P_{2^{2 m}-1}(x) \equiv 1+2 S_{m-1}^{o}(x)(\bmod 4) ;(m \geq 1)$

2) $P_{2^{2 m+1}-1}(x) \equiv 1+2 x\left(1-\delta_{m, 0}\right)+2 S_{m}^{e}(x)(\bmod 4) ;(m \geq 0)$

3) $P_{2^{2 m}-2}(x) \equiv 1+x^{-1} S_{2 m-1}(x)^{2}-2 S_{m}^{e}(x)(\bmod 4) ;(m \geq 1)$

4) $P_{2^{2 m+1}-2}(x) \equiv 1+x^{-1} S_{2 m}(x)^{2}-2 S_{m}^{o}(x)(\bmod 4) ;(m \geq 0)$

5) $Q_{2^{2 m}-1}(x) \equiv 1-x+2 x^{2^{2 m-1}}-S_{2 m-1}(x)^{2}+2 x S_{m}^{e}(x)(\bmod 4) ;(m \geq 1)$

6) $Q_{2^{2 m+1}-1}(x) \equiv 1-x+2 x^{2^{2 m}}\left(1-\delta_{m, 0}\right)-S_{2 m}(x)^{2}+2 x S_{m}^{o}(x)(\bmod 4) ;(m \geq 0)$

7) $Q_{2^{2 m}-2}(x) \equiv 1+2 S_{2 m-1}(x)(\bmod 4) ;(m \geq 1)$

8) $Q_{2^{2 m+1}-2}(x) \equiv 1+2 x\left(1-\delta_{m, 0}\right)+2 S_{2 m}(x)(\bmod 4) .(m \geq 0)$

Proof. We prove this result by induction on

$$
n \in\left\{2^{k}-1 \mid k\right\} \cup\left\{2^{k}-2 \mid k\right\} .
$$

When we compute $P_{n}(x)$ or $Q_{n}(x)$, the induction hypothesis is that the expressions for $P_{\ell}(x)$ and $Q_{\ell}(x)$ are true for $\ell<n$ and $\ell \in\left\{2^{k}-1 \mid k\right\} \cup\left\{2^{k}-2 \mid k\right\}$. Relations 1) - 8) are true for $m=0$ or $m=1$. In the sequel let $m \geq 2$.

1) Using the induction hypothesis, we have

$$
\begin{aligned}
Q_{2^{2 m-1}-1}(-x) & \equiv 1+x+2 x^{2^{2 m-2}}-S_{2 m-2}(x)^{2}-2 x S_{m-1}^{o}(x) ; \\
P_{2^{2 m-1}-1}(x) & \equiv 1+2 x+2 S_{m-1}^{e}(x) ; \\
P_{2^{2 m-1}-1}(-x) & \equiv 1-2 x+2 S_{m-1}^{e}(x) ; \\
P_{2^{2 m-1}-2}(x) & \equiv 1+x^{-1} S_{2 m-2}(x)^{2}-2 S_{m-1}^{o}(x) .
\end{aligned}
$$

By Lemma 3.1, we obtain

$$
\begin{aligned}
P_{2^{2 m}-1} \equiv & Q_{2^{2 m-1}-1}(-x) P_{2^{2 m-1}-1}(x)-x P_{2^{2 m-1}-1}(-x) P_{2^{2 m-1}-2}(x) \\
\equiv & \left(1+2 x+2 S_{m-1}^{e}(x)\right) \\
& \times\left(\left(1+x+2 x^{2^{2 m-2}}-S_{2 m-2}(x)^{2}-2 x S_{m-1}^{o}(x)\right)\right. \\
& \left.\quad-x\left(1+x^{-1} S_{2 m-2}(x)^{2}-2 S_{m-1}^{o}(x)\right)\right) \\
\equiv & \left(1+2 x+2 S_{m-1}^{e}(x)\right)\left(1+2 x^{2^{2 m-2}}-2 S_{2 m-2}(x)^{2}\right) \\
\equiv & 1+2 x+2 S_{m-1}^{e}(x)+2 x^{2^{2 m-2}}-2 S_{2 m-2}(x)^{2} \\
\equiv & 1+2 S_{m-1}^{e}(x)+2 x^{2^{2 m-2}}-2 S_{2 m-1}(x) \\
\equiv & 1+2 S_{m-1}^{o}(x) .
\end{aligned}
$$

2) Using the induction hypothesis, we have

$$
Q_{2^{2 m}-1}(-x) \equiv 1+x+2 x^{2^{2 m-1}}-S_{2 m-1}(x)^{2}+2 x S_{m}^{e}(x) ;
$$




$$
\begin{aligned}
P_{2^{2 m}-1}(x) & \equiv 1+2 S_{m-1}^{o}(x) ; \\
P_{2^{2 m}-1}(-x) & \equiv 1+2 S_{m-1}^{o}(x) ; \\
P_{2^{2 m}-2}(x) & \equiv 1+x^{-1} S_{2 m-1}(x)^{2}-2 S_{m}^{e}(x) .
\end{aligned}
$$

By Lemma 3.1, we obtain

$$
\begin{aligned}
P_{2^{2 m+1}-1} \equiv & Q_{2^{2 m}-1}(-x) P_{2^{2 m}-1}(x)-x P_{2^{2 m}-1}(-x) P_{2^{2 m}-2}(x) \\
\equiv & \left(1+2 S_{m-1}^{o}(x)\right) \\
& \times\left(\left(1+x+2 x^{2^{2 m-1}}-S_{2 m-1}(x)^{2}+2 x S_{m}^{e}(x)\right)\right. \\
& \left.\quad-x\left(1+x^{-1} S_{2 m-1}(x)^{2}-2 S_{m}^{e}(x)\right)\right) \\
\equiv & \left(1+2 S_{m-1}^{o}(x)\right)\left(1+2 x^{2^{2 m-1}}-2 S_{2 m-1}(x)^{2}\right) \\
\equiv & 1+2 S_{m-1}^{o}(x)+2 x^{2^{2 m-1}}-2 S_{2 m-1}(x)^{2} \\
\equiv & 1+2 S_{m-1}^{o}(x)+2 x^{2^{2 m-1}}-2 S_{2 m}(x)+2 x \\
\equiv & 1+2 x+2 S_{m}^{e}(x) .
\end{aligned}
$$

3) Using the induction hypothesis, we have

$$
\begin{aligned}
Q_{2^{2 m-1}-2}(x) & \equiv 1+2 x+2 S_{2 m-2} \\
P_{2^{2 m-1}-1}(x) & \equiv 1+2 x+2 S_{m-1}^{e} \\
P_{2^{2 m-1}-2}(x) & \equiv 1+x^{-1} S_{2 m-2}^{2}-2 S_{m-1}^{o} \\
P_{2^{2 m-1}-2}(-x) & \equiv 1-x^{-1} S_{2 m-2}^{2}-2 S_{m-1}^{o} .
\end{aligned}
$$

By Lemma 3.1, we obtain

$$
\begin{aligned}
P_{2^{2 m}-2}(x) \equiv & Q_{2^{2 m-1}-2}(-x) P_{2^{2 m-1}-1}(x)-x P_{2^{2 m-1}-2}(-x) P_{2^{2 m-1}-2}(x) \\
\equiv & \left(1+2 x+2 S_{2 m-2}\right)\left(1+2 x+2 S_{m-1}^{e}\right) \\
& -x\left(1+x^{-1} S_{2 m-2}^{2}-2 S_{m-1}^{o}\right)\left(1-x^{-1} S_{2 m-2}^{2}-2 S_{m-1}^{o}\right) \\
\equiv & 1+2 S_{2 m-2}+2 S_{m-1}^{e}-x\left(1-2 S_{m-1}^{o}\right)^{2}+x\left(x^{-1} S_{2 m-2}^{2}\right)^{2} \\
\equiv & 1+2 S_{2 m-2}+2 S_{m-1}^{e}-x+x^{-1}\left(S_{2 m-1}-x\right)^{2} \\
\equiv & 1+x^{-1} S_{2 m-1}^{2}+2 S_{m}^{e} .
\end{aligned}
$$

4) Using the induction hypothesis, we have

$$
\begin{aligned}
P_{2^{2 m}-1}(x) & \equiv 1+2 S_{m-1}^{o}(x) ; \\
P_{2^{2 m}-2}(x) & \equiv 1+x^{-1} S_{2 m-1}(x)^{2}-2 S_{m}^{e}(x) ; \\
Q_{2^{2 m}-2}(x) & \equiv 1+2 S_{2 m-1}(x) .
\end{aligned}
$$

By Lemma 3.1, we obtain

$$
\begin{aligned}
P_{2^{2 m+1}-2}(x) \equiv & Q_{2^{2 m}-2}(-x) P_{2^{2 m}-1}(x)-x P_{2^{2 m}-2}(-x) P_{2^{2 m}-2}(x) \\
\equiv & \left(1+2 S_{2 m-1}(-x)\right)\left(1+2 S_{m-1}^{o}(x)\right) \\
& -x\left(1-x^{-1} S_{2 m-1}(-x)^{2}-2 S_{m}^{e}(-x)\right)\left(1+x^{-1} S_{2 m-1}(x)^{2}-2 S_{m}^{e}(x)\right) \\
\equiv & 1+2 S_{2 m-1}(x)+2 S_{m-1}^{o}(x)-x+x^{-1} S_{2 m-1}(x)^{4} \\
\equiv & 1+2 S_{2 m-1}(x)+2 S_{m-1}^{o}(x)-x+x^{-1}\left(S_{2 m}(x)^{2}-2 x S_{2 m}(x)+x^{2}\right) \\
\equiv & 1+x^{-1} S_{2 m}(x)^{2}-2 S_{m}^{o}(x) .
\end{aligned}
$$


5) Using the induction hypothesis, we have

$$
\begin{aligned}
& P_{2^{2 m-1}-1}(x) \equiv 1+2 x+2 S_{m-1}^{e}(x) \\
& Q_{2^{2 m-1}-1}(x) \equiv 1-x+2 x^{2^{2 m-2}}-S_{2 m-2}(x)^{2}+2 x S_{m-1}^{o}(x) ; \\
& Q_{2^{2 m-1}-2}(x) \equiv 1+2 x+2 S_{2 m-2}(x) .
\end{aligned}
$$

By Lemma 3.1, we obtain

$$
\begin{aligned}
Q_{2^{2 m}-1}(x) \equiv & Q_{2^{2 m-1}-1}(-x) Q_{2^{2 m-1}-1}(x)-x P_{2^{2 m-1}-1}(-x) Q_{2^{2 m-1}-2}(x) \\
\equiv & \left(1+x+2 x^{2^{2 m-2}}-S_{2 m-2}^{2}+2 x S_{m-1}^{o}\right) \\
& \left(1-x+2 x^{2^{2 m-2}}-S_{2 m-2}^{2}+2 x S_{m-1}^{o}\right) \\
& -x\left(1+2 x+2 S_{m-1}^{e}\right)\left(1+2 x+2 S_{2 m-2}\right) \\
\equiv & \left(1-S_{2 m-2}^{2}\right)^{2}-x^{2}-\left(x+2 x S_{m-1}^{e}+2 x S_{2 m-2}\right) \\
\equiv & 1+2 S_{2 m-2}^{2}+S_{2 m-1}^{2}+2 x S_{2 m-1}-\left(x+2 x S_{m-1}^{e}+2 x S_{2 m-2}\right) \\
\equiv & 1-x+2 x^{2^{2 m-1}}-S_{2 m-1}^{2}+2 x S_{m}^{e} .
\end{aligned}
$$

6 ) Using the induction hypothesis, we have

$$
\begin{aligned}
& P_{2^{2 m}-1}(x) \equiv 1+2 S_{m-1}^{o}(x) ; \\
& Q_{2^{2 m}-1}(x) \equiv 1-x+2 x^{2^{2 m-1}}-S_{2 m-1}(x)^{2}+2 x S_{m}^{e}(x) ; \\
& Q_{2^{2 m}-2}(x) \equiv 1+2 S_{2 m-1}(x) .
\end{aligned}
$$

By Lemma 3.1, we obtain

$$
\begin{aligned}
Q_{2^{2 m+1}-1}(x) \equiv & Q_{2^{2 m}-1}(-x) Q_{2^{2 m}-1}(x)-x P_{2^{2 m}-1}(-x) Q_{2^{2 m}-2}(x) \\
\equiv & \left(1+x+2 x^{2^{2 m-1}}-S_{2 m-1}^{2}+2 x S_{m}^{e}\right) \\
\equiv & \left(1-x+2 x^{2^{2 m-1}}-S_{2 m-1}^{2}+2 x S_{m}^{e}\right) \\
& -x\left(1+2 S_{m-1}^{o}\right)\left(1+2 S_{2 m+1}\right) \\
\equiv & 1+3 S_{2 m}^{2}+2 x S_{2 m}+2 x^{2^{2 m}}-x+2 x S_{m-1}^{o}+2 x S_{2 m-1} \\
\equiv & 1-x+2 x^{2^{2 m}}-S_{2 m}^{2}+2 x S_{m}^{o} .
\end{aligned}
$$

7) Using the induction hypothesis, we have

$$
\begin{aligned}
& P_{2^{2 m-1}-2}(x) \equiv 1+x^{-1} S_{2 m-2}(x)^{2}-2 S_{m-1}^{o}(x) ; \\
& Q_{2^{2 m-1}-1}(x) \equiv 1-x+2 x^{2^{2 m-2}}-S_{2 m-2}(x)^{2}+2 x S_{m-1}^{o}(x) ; \\
& Q_{2^{2 m-1}-2}(x) \equiv 1+2 x+2 S_{2 m-2}(x) .
\end{aligned}
$$

By Lemma 3.1, we obtain

$$
\begin{aligned}
& Q_{2^{2 m}-2}(x) \\
\equiv & Q_{2^{2 m-1}-2}(-x) Q_{2^{2 m-1}-1}(x)-x P_{2^{2 m-1}-2}(-x) Q_{2^{2 m-1}-2}(x) \\
\equiv & \left(1+2 x+2 S_{2 m-2}\right)\left(1-x+2 x^{2^{2 m-2}}-S_{2 m-2}^{2}+2 x S_{m-1}^{o}\right) \\
& -x\left(1-x^{-1} S_{2 m-2}^{2}+2 S_{m-1}^{o}\right)\left(1+2 x+2 S_{2 m-2}\right) \\
\equiv & 1+x+2 x 2^{2 m-2}-S_{2 m-2}^{2}+2 x S_{m-1}^{o}+2 x^{2}+2 x S_{2 m-2}^{2}+2 S_{2 m-2}+2 x S_{2 m-2} \\
& +2 S_{2 m-2}^{3}-x\left(1+2 x+2 S_{2 m-2}-x^{-1} S_{2 m-2}^{2}+2 S_{2 m-2}^{2}+2 x^{-1} S_{2 m-2}^{3}+2 S_{m-1}^{o}\right)
\end{aligned}
$$


$\equiv 1+2 S_{2 m-1}$.

8) Using the induction hypothesis, we have

$$
\begin{aligned}
& P_{2^{2 m}-2}(x) \equiv 1+x^{-1} S_{2 m-1}(x)^{2}-2 S_{m}^{e}(x) ; \\
& Q_{2^{2 m}-1}(x) \equiv 1-x+2 x^{2^{2 m-1}}-S_{2 m-1}(x)^{2}+2 x S_{m}^{e}(x) ; \\
& Q_{2^{2 m}-2}(x) \equiv 1+2 S_{2 m-1}(x) ;
\end{aligned}
$$

By Lemma 3.1, we obtain

$$
\begin{aligned}
Q_{2^{2 m+1}-2}(x) \equiv & Q_{2^{2 m}-2}(-x) Q_{2^{2 m}-1}(x)-x P_{2^{2 m}-2}(-x) Q_{2^{2 m}-2}(x) \\
\equiv & \left(1+2 S_{2 m-1}\right)\left(1-x+2 x^{2^{2 m-1}}-S_{2 m-1}^{2}+2 x S_{m}^{e}\right) \\
& -x\left(1-x^{-1} S_{2 m-1}^{2}+2 S_{m}^{e}\right)\left(1+2 S_{2 m-1}\right) \\
\equiv & 1-x+2 x^{2^{2 m-1}}-S_{2 m-1}^{2}+2 x S_{m}^{e}+2 S_{2 m-1}+2 x S_{2 m-1}+2 S_{2 m-1}^{3} \\
& -\left(x-S_{2 m-1}^{2}+2 x S_{m}^{e}+2 x S_{2 m-1}+2 S_{2 m-1}^{3}\right) \\
\equiv & 1+2 x+2 S_{2 m-1}+2 x^{2^{2 m-1}} \\
\equiv & 1+2 x+2 S_{2 m} .
\end{aligned}
$$

The explicit expressions of $P_{2^{2 m}-2}(x)$ and $P_{2^{2 m}}-2(x)$ give the explicit expression for $C(x)$.

Proposition 3.3.

$$
C(x) \equiv 1-x^{-1} S_{\infty}(x)^{2}+2 S_{\infty}^{e}(x) \quad(\bmod 4) .
$$

Proof. By Theorem 2.1,

$$
C(x)=\lim _{m \rightarrow \infty} P_{2^{2 m}-2}(x) / Q_{2^{2 m}-2}(x) .
$$

The constant term of $Q_{2^{2 m}+2}(x)$ being 1 , its inverse $1 / Q_{2^{2 m}+2}(x)$ belongs to $\mathbb{Z}[[x]]$. By 3) and 7) of Proposition 3.2,

$$
\begin{aligned}
C(x) & \equiv \lim _{m \rightarrow \infty} \frac{1+x^{-1} S_{2 m-1}(x)^{2}-2 S_{m}^{e}(x)}{1+2 S_{2 m-1}(x)} \\
& \equiv \lim _{m \rightarrow \infty}\left(1+x^{-1} S_{2 m-1}(x)^{2}-2 S_{m}^{e}(x)\right)\left(1+2 S_{2 m-1}(x)\right) \\
& \equiv \lim _{m \rightarrow \infty} 1+x^{-1} S_{2 m-1}(x)^{2}-2 S_{m}^{e}(x)+2 S_{2 m-1}(x)+2 x^{-1} S_{2 m-1}(x)^{3} \\
& \equiv 1+x^{-1} S_{\infty}(x)^{2}+2 S_{\infty}^{e}(x)+2 S_{\infty}(x)+2 x^{-1} S_{\infty}(x)^{3} \\
& \equiv 1+x^{-1} S_{\infty}(x)^{2}+2 S_{\infty}^{e}(x)+2 S_{\infty}(x)+2 x^{-1} S_{\infty}(x)\left(S_{\infty}(x)-x\right) \\
& \equiv 1-x^{-1} S_{\infty}(x)^{2}+2 S_{\infty}^{e}(x)
\end{aligned}
$$

Now we prove Theorem 1.4 by repeated applying the Cartier operators to the right hand side of congruence (3.1).

Proof of Theorem 1.4. We indicate the canonical projection of $\mathbb{Z}$ onto $\mathbb{Z} / 4 \mathbb{Z}$ with a bar over the series. First notice that the following identities hold:

$$
\begin{aligned}
\Lambda_{0} \bar{S}_{\infty}(x) & =\bar{S}_{\infty}(x), & \Lambda_{1} \bar{S}_{\infty}(x) & =1, \\
\Lambda_{0} \bar{S}_{\infty}^{e}(x) & =\bar{S}_{\infty}^{o}(x), & \Lambda_{1} \bar{S}_{\infty}^{e}(x) & =1, \\
\Lambda_{0} \bar{S}_{\infty}^{o}(x) & =\bar{S}_{\infty}^{e}(x), & \Lambda_{1} \bar{S}_{\infty}^{o}(x) & =0, \\
\Lambda_{0} x^{-1} \bar{S}_{\infty}^{2}(x) & =2 \bar{S}_{\infty}(x), & \Lambda_{1} x^{-1} \bar{S}_{\infty}^{2}(x) & =1+x^{-1} \bar{S}_{\infty}^{2}(x) .
\end{aligned}
$$


We apply $\Lambda_{0}$ and $\Lambda_{1}$ repeatedly to $\bar{C}(x)$ and obtain a finite number of series:

$$
\begin{aligned}
& \bar{C}(x)=1-x^{-1} \bar{S}_{\infty}(x)^{2}+2 \bar{S}_{\infty}^{e}(x)=: f_{0}, \\
& \Lambda_{0} f_{0}=1-2 \bar{S}_{\infty}(x)+2 \bar{S}_{\infty}^{o}(x)=1+2 \bar{S}_{\infty}^{e}(x)=: f_{1}, \\
& \Lambda_{1} f_{0}=-x^{-1} \bar{S}_{\infty}(x)^{2}+1=: f_{2}, \\
& \Lambda_{0} f_{1}=1+2 \bar{S}_{\infty}^{o}(x)=: f_{3}, \\
& \Lambda_{1} f_{1}=2=: f_{4} \\
& \Lambda_{0} f_{2}=2 \bar{S}_{\infty}(x)+1=: f_{5}, \\
& \Lambda_{1} f_{2}=-1-x^{-1} \bar{S}_{\infty}(x)^{2}=: f_{6}, \\
& \Lambda_{0} f_{3}=1+2 \bar{S}_{\infty}^{e}(x)=f_{1}, \\
& \Lambda_{1} f_{3}=0=: f_{7}, \\
& \Lambda_{0} f_{4}=2=f_{4}, \\
& \Lambda_{1} f_{4}=0=f_{7}, \\
& \Lambda_{0} f_{5}=2 \bar{S}_{\infty}(x)+1=f_{5}, \\
& \Lambda_{1} f_{5}=2=f_{4}, \\
& \Lambda_{0} f_{6}=-1+2 \bar{S}_{\infty}(x)=: f_{8}, \\
& \Lambda_{1} f_{6}=-1-x^{-1} \bar{S}_{\infty}(x)^{2}=f_{6}, \\
& \Lambda_{0} f_{8}=-1+2 \bar{S}_{\infty}(x)=f_{8}, \\
& \Lambda_{1} f_{8}=2=f_{4} .
\end{aligned}
$$

We see from the computation above that the 2-kernel of $\bar{C}(x)$ consists of 9 elements, $f_{0}$ through $f_{8}$. The structure of the 2-kernel is

$$
[[1,2],[3,4],[5,6],[1,7],[4,7],[5,4],[8,6],[7,7],[8,4]] .
$$

The following lemma is used in the proof of Theorem 1.1 (see, for example, [22]).

\section{Lemma 3.4.}

$$
\sqrt{1-4 x} \equiv 1+2 \sum_{k=0}^{\infty} x^{2^{k}} \quad(\bmod 4) .
$$

Proof of Theorem 1.1. By Proposition 3.3 we know that

$$
C(x) \equiv 1-x^{-1} S_{\infty}(x)^{2}+2 S_{\infty}^{e}(x) \quad(\bmod 4) .
$$

Therefore, we only need to evaluate $\left(S_{\infty}(x)\right)^{2}(\bmod 4)$ and $S_{\infty}^{e}(x)(\bmod 2)$. By Lemma 3.4,

$$
S_{\infty}(x) \equiv \frac{1-\sqrt{1-4 x}}{2} \quad(\bmod 2)
$$

so that

$$
S_{\infty}(x)^{2} \equiv\left(\frac{1-\sqrt{1-4 x}}{2}\right)^{2} \equiv \frac{1-2 x-\sqrt{1-4 x}}{2} \quad(\bmod 4) .
$$

To calculate $S_{\infty}^{e}(x)(\bmod 2)$, we notice that

$$
\begin{aligned}
S_{\infty}^{e}(x)^{2}+S_{\infty}^{e}(x) & =S_{\infty}^{e}\left(x^{2}\right)+S_{\infty}^{e}(x)+2 x \psi(x) \\
& =S_{\infty}(x)+2 x \psi(x)
\end{aligned}
$$




$$
=\frac{1-\sqrt{1-4 x}}{2}+2 x \xi(x)+2 x \psi(x),
$$

where

$$
\psi(x)=\frac{1}{2 x}\left(S_{\infty}^{e}(x)^{2}-S_{\infty}^{e}\left(x^{2}\right)\right) \quad \text { and } \quad \xi(x)=\frac{1}{2 x}\left(S_{\infty}(x)-\frac{1-\sqrt{1-4 x}}{2}\right)
$$

are in $\mathbb{Z}[[x]]$. We remark that by Lemma 3.4 , if

$$
f(x), g(x) \in \mathbb{Z}[[x]] \quad \text { and } \quad f(x) \equiv g(x) \quad(\bmod 2),
$$

then

Therefore

$$
\sqrt{1+4 x f(x)} \equiv \sqrt{1+4 x g(x)} \quad(\bmod 4) .
$$

Finally

$$
\begin{aligned}
C(x) & \equiv 1-x^{-1} S_{\infty}(x)^{2}+2 S_{\infty}^{e}(x) \\
& \equiv \frac{\sqrt{1-4 x}-1}{2 x}+1+\sqrt{2 \sqrt{1-4 x}-1} \quad(\bmod 4) .
\end{aligned}
$$

\section{Period-Doubling CONTINUed Fraction}

In this section we prove Theorem 1.2 using Theorem 1.1 and Theorem 2.3. As a corollary, we get the explicit expression of $\bar{D}(x)$ as a power series and from this we calculate the 2 -kernel of the sequence $\left(\bar{d}_{n}\right)$.

Proof of Theorem 1.2. In Theorem 2.3, if we let

$$
\begin{aligned}
u_{1} & =t_{1}=-1 ; \\
u_{n} & =t_{2 n-2}+t_{2 n-1}=0 ; \quad(n \geq 2) \\
v_{0} & =t_{0}=1 ; \\
v_{n} & =t_{2 n-1} t_{2 n}=-t_{n-1} t_{n}=s_{n-1}, \quad(n \geq 1)
\end{aligned}
$$

we get

$$
C(x)=\frac{t_{0}}{1+\frac{t_{1} x}{1+\frac{t_{2} x}{1+\frac{t_{3} x}{1+\frac{t_{4} x}{\ddots}}}}}=\frac{1}{1-x-\frac{s_{0} x^{2}}{1-\frac{s_{1} x^{2}}{1-\frac{s_{2} x^{2}}{\ddots}}}}=\frac{1}{1-x-x^{2} D\left(-x^{2}\right)} .
$$

We define

$$
\begin{aligned}
& H_{1}(x)=\frac{\sqrt{1-4 x}-1}{2 x}+1+\sqrt{2 \sqrt{1-4 x}-1}=1-3 x+\cdots \\
& H_{2}(x)=\frac{1+\sqrt{1+4 x}}{2}=1+x+\cdots
\end{aligned}
$$




$$
H_{3}(x)=\sqrt{2 \sqrt{1-4 x^{2}}-1}=1-2 x^{2}+\cdots
$$

Then our goal (1.4) can be written as

$$
D(x) \equiv \frac{H_{1}(x) H_{3}(x)-1}{x} \quad(\bmod 4) .
$$

Since $C(x) \equiv H_{1}(x)(\bmod 4)$ and the constant term of $C(x)$ is 1 , by Eq. (4.1) we know that

$$
-x^{2} D\left(-x^{2}\right)=\frac{1}{C(x)}-(1-x) \equiv \frac{1}{H_{1}(x)}-1+x \quad(\bmod 4) .
$$

We only need to show that

$$
\frac{1}{H_{1}(x)}-1+x \equiv-x^{2} \times \frac{H_{2}\left(-x^{2}\right) H_{3}\left(-x^{2}\right)-1}{-x^{2}} \quad(\bmod 4),
$$

that is,

$$
\frac{1}{H_{1}(x)}+x \equiv H_{2}\left(-x^{2}\right) H_{3}\left(-x^{2}\right) \quad(\bmod 4) .
$$

Since the constant term of $H_{1}(x)$ is 1 , this is equivalent to

$$
H_{1}(x)\left(H_{2}\left(-x^{2}\right) H_{3}\left(-x^{2}\right)-x\right) \equiv 1 \quad(\bmod 4) .
$$

By congruences (3.3), (3.4) and (3.2),

$$
\begin{aligned}
H_{1}(x) & \equiv-\frac{S_{\infty}(x)^{2}}{x}+2 S_{\infty}^{e}+1 \quad(\bmod 4), \\
H_{2}\left(-x^{2}\right) & =\frac{1+\sqrt{1-4 x^{2}}}{2} \\
& \equiv 1-x^{2}-S_{\infty}\left(x^{2}\right)^{2} \\
& \equiv 1-x^{2}-\left(S_{\infty}(x)-x\right)^{2} \\
& \equiv 1+(2 x-1) S_{\infty}(x)^{2} \quad(\bmod 4), \\
H_{3}\left(-x^{2}\right) & =\sqrt{2 \sqrt{1-4 x^{4}}-1} \\
& \equiv 1+2 S_{\infty}^{e}\left(x^{4}\right) \\
& \equiv 1-2 x+2 S_{\infty}^{e}(x) \quad(\bmod 4) .
\end{aligned}
$$

Taking account of the above congruence relations and after rearranging the terms, we get

$$
\begin{aligned}
& x H_{1}(x)\left(H_{2}\left(-x^{2}\right) H_{3}\left(-x^{2}\right)-x\right) \\
\equiv & x-x S_{\infty}(x)^{2}+x^{2}-S_{\infty}(x)^{2}\left(1-S_{\infty}(x)^{2}+x\right) \\
& +S_{\infty}^{e}(x)\left(2 x^{2}+2 S_{\infty}(x)^{2}\left(1-S_{\infty}(x)^{2}\right)\right) \quad(\bmod 4) .
\end{aligned}
$$

Since

$$
\begin{aligned}
2 S_{\infty}(x)^{2}\left(1-S_{\infty}(x)^{2}\right) & \equiv 2\left(S_{\infty}(x)-x\right)\left(1-S_{\infty}(x)+x\right) \\
& \equiv 2\left(S_{\infty}(x)-S_{\infty}(x)^{2}-x-x^{2}\right) \\
& \equiv 2 x^{2} \quad(\bmod 4),
\end{aligned}
$$

and

$$
S_{\infty}(x)^{4} \equiv\left(S_{\infty}(x)-x\right)^{2}
$$




$$
\equiv S_{\infty}(x)^{2}+x^{2}-2 x S_{\infty}(x) \quad(\bmod 4),
$$

congruence (4.2) becomes

$$
\begin{aligned}
& x H_{1}(x)\left(H_{2}\left(-x^{2}\right) H_{3}\left(-x^{2}\right)-x\right) \\
\equiv & x-x S_{\infty}(x)^{2}+x^{2}-S_{\infty}(x)^{2}\left(1-S_{\infty}(x)^{2}+x\right) \\
\equiv & x+2 x^{2}+2 x S_{\infty}(x)^{2}+2 x S_{\infty}(x) \\
\equiv & x \quad(\bmod 4) .
\end{aligned}
$$

From Theorem 1.2 we obtain the following explicit expression for $D(x)(\bmod 4)$.

\section{Corollary 4.1.}

$$
D(x) \equiv 1-x^{-1} S_{\infty}(x)^{2}+2 x^{-1} S_{\infty}^{o}(x)\left(1+S_{\infty}(x)\right) \quad(\bmod 4) .
$$

Proof of Corollary 4.1. We obtained the following congruence in Theorem 1.2,

$$
D(x) \equiv \frac{(1+\sqrt{1+4 x}) \sqrt{2 \sqrt{1-4 x^{2}}-1}-2}{2 x} \quad(\bmod 4) .
$$

From congruence (3.3) we know

$$
\begin{aligned}
\frac{1+\sqrt{1+4 x}}{2} & \equiv 1+x-S_{\infty}(-x)^{2} \quad(\bmod 4) \\
& \equiv 1+x-S_{\infty}(x)^{2} \quad(\bmod 4)
\end{aligned}
$$

By Eq. (3.4)

$$
\sqrt{2 \sqrt{1-4 x^{2}}-1} \equiv 1+2 S_{\infty}^{o}(x) \quad(\bmod 4) .
$$

Therefore

$$
\begin{aligned}
D(x) & \equiv \frac{\left(1+x-S_{\infty}(x)^{2}\right)\left(1+2 S_{\infty}^{o}(x)\right)-1}{x} \\
& \equiv 1-x^{-1} S_{\infty}(x)^{2}+2 x^{-1} S_{\infty}^{o}(x)\left(1+S_{\infty}(x)\right) \quad(\bmod 4) .
\end{aligned}
$$

Proof of Theorem 1.5. By Theorem 1.2 and Theorem 1.3 we know that the sequence $\left(\bar{d}_{n}\right)$ is 2-automatic. By Corollary 4.1 ,

$$
\bar{D}(x)=1-x^{-1} \bar{S}_{\infty}(x)^{2}+2 x^{-1} \bar{S}_{\infty}^{o}(x)+2 x^{-1} \bar{S}_{\infty}^{o}(x) \bar{S}_{\infty}(x) .
$$

We compute the 2-kernel of $\bar{D}(x)$. First we notice that

$$
\begin{array}{rlrl} 
& \Lambda_{0} 2 x^{-1} \bar{S}_{\infty}^{o}(x) \bar{S}_{\infty}(x) & \Lambda_{1} 2 x^{-1} \bar{S}_{\infty}^{o}(x) \bar{S}_{\infty}(x) \\
= & \Lambda_{0}\left(2 \sum_{k, j=0}^{\infty} x^{2^{2 k}+2^{j}-1}\right) & = & \Lambda_{1}\left(2 x \sum_{k=0, j=1}^{\infty} x^{2^{2 k+1}+2^{j}-2}\right) \\
= & \Lambda_{0}\left(2 \sum_{k=0}^{\infty} x^{2^{2 k+1}}\right) & = & \Lambda_{1}\left(2 x \sum_{k=0, j=1}^{\infty}\left(x^{2}\right)^{2^{2 k}+2^{j-1}-1}\right) \\
= & \Lambda_{0}\left(2 \sum_{k=0}^{\infty}\left(x^{2}\right)^{2^{2 k}}\right) & = & 2 \sum_{k, j=0}^{\infty} x^{2^{2 k}+2^{j}-1} \\
= & 2 \bar{S}_{\infty}^{e}(x), & = & 2 x^{-1} \bar{S}_{\infty}^{e}(x) \bar{S}_{\infty}(x) .
\end{array}
$$

So that

$$
\bar{D}(x)=1-x^{-1} \bar{S}_{\infty}(x)^{2}+2 x^{-1} \bar{S}_{\infty}^{o}(x)+2 x^{-1} \bar{S}_{\infty}^{o}(x) \bar{S}_{\infty}(x)=: f_{0},
$$




$$
\begin{aligned}
& \Lambda_{0}\left(f_{0}\right)=1+2 \bar{S}_{\infty}(x)+2 \bar{S}_{\infty}^{e}(x)=1+2 \bar{S}_{\infty}^{o}(x)=: f_{1}, \\
& \Lambda_{1}\left(f_{0}\right)=-1-x^{-1} \bar{S}_{\infty}(x)^{2}+2 x^{-1} \bar{S}_{\infty}^{e}(x)+2 x^{-1} \bar{S}_{\infty}^{e}(x) \bar{S}_{\infty}(x)=f_{0} .
\end{aligned}
$$

The last equality holds because

$$
\begin{aligned}
& 1-x^{-1} \bar{S}_{\infty}(x)^{2}+2 x^{-1} \bar{S}_{\infty}^{o}(x)+2 x^{-1} \bar{S}_{\infty}^{o}(x) \bar{S}_{\infty}(x) \\
- & \left(-1-x^{-1} \bar{S}_{\infty}(x)^{2}+2 x^{-1} \bar{S}_{\infty}^{e}(x)+2 x^{-1} \bar{S}_{\infty}^{e}(x) \bar{S}_{\infty}(x)\right) \\
= & 2+2 x^{-1} \bar{S}_{\infty}^{o}(x)+2 x^{-1} \bar{S}_{\infty}^{o}(x) \bar{S}_{\infty}(x)+2 x^{-1} \bar{S}_{\infty}^{e}(x)+2 x^{-1} \bar{S}_{\infty}^{e}(x) \bar{S}_{\infty}(x) \\
= & 2+2 x^{-1} \bar{S}_{\infty}(x)+2 x^{-1} \bar{S}_{\infty}(x)^{2} \\
= & 0 .
\end{aligned}
$$

To continue,

$$
\begin{aligned}
& \Lambda_{0}\left(f_{1}\right)=1+2 \bar{S}_{\infty}^{e}(x)=: f_{2}, \\
& \Lambda_{1}\left(f_{1}\right)=0=: f_{3}, \\
& \Lambda_{0}\left(f_{2}\right)=1+2 \bar{S}_{\infty}^{o}(x)=f_{1}, \\
& \Lambda_{1}\left(f_{2}\right)=2=: f_{4} .
\end{aligned}
$$

We see that the structure of the 2 -kernel of $\left(\bar{d}_{n}\right)$ is $[[1,0],[2,3],[1,4],[3,3],[4,3]]$.

\section{Proof of Theorem 1.6}

In this section we prove Theorem 1.6. First we recall that from Theorem 1.1 and 1.2 that

where

$$
\begin{aligned}
& C(x) \equiv \varphi(x) \quad(\bmod 4), \\
& D(x) \equiv \psi(x) \quad(\bmod 4),
\end{aligned}
$$

$$
\begin{aligned}
& \varphi(x)=\frac{\sqrt{1-4 x}-1}{2 x}+1+\sqrt{2 \sqrt{1-4 x}-1} \in \mathbb{Z}[[x]], \\
& \psi(x)=\frac{(1+\sqrt{1+4 x}) \sqrt{2 \sqrt{1-4 x^{2}}-1}-2}{2 x} \in \mathbb{Z}[[x]] .
\end{aligned}
$$

By rearranging the terms and squaring both sides of the equalities, we obtain annihilating polynomials $P(x, y)$ and $Q(x, y)$ of $\varphi(x)$ and $\psi(x)$ respectively:

$$
\begin{aligned}
P(x, y)= & y^{4} x^{2}-4 y^{3} x^{2}+2 y^{3} x^{1}+8 y^{2} x^{2}-4 y^{2} x^{1}+8 y x^{2} \\
& +16 x^{3}+y^{2}-16 x^{2}+8 x^{1}-1 \\
Q(x, y)= & y^{8} x^{7}+8 y^{7} x^{6}+4 y^{6} x^{6}+30 y^{6} x^{5}+32 y^{4} x^{7}+24 y^{5} x^{5} \\
& +64 y^{4} x^{6}+68 y^{5} x^{4}+14 y^{4} x^{5}+128 y^{3} x^{6}+48 y^{4} x^{4} \\
& +256 y^{3} x^{5}+64 y^{2} x^{6}+97 y^{4} x^{3}+56 y^{3} x^{4}+224 y^{2} x^{5} \\
& +256 x^{7}+32 y^{3} x^{3}+372 y^{2} x^{4}+128 y x^{5}+84 y^{3} x^{2}+78 y^{2} x^{3} \\
& +192 y x^{4}-96 x^{5}-12 y^{2} x^{2}+232 y x^{3}+64 x^{4}+40 y^{2} x \\
& +44 y x^{2}+73 x^{3}-24 y x+52 x^{2}+8 y+8 x-8 .
\end{aligned}
$$

For $n \in \mathbb{N}^{*}$, we let $\pi_{n}$ denote the canonical projection of $\mathbb{Z}$ onto $\mathbb{Z} / n \mathbb{Z}$, and by abuse of notation, the canonical projection of $\mathbb{Z}[[x]]$ onto $\mathbb{Z} / n \mathbb{Z}[[x]]$, of $\mathbb{Z}[x, y]$ onto $\mathbb{Z} / n \mathbb{Z}[x, y]$, etc. 
Since $P(x, \varphi(x))=0, Q(x, \psi(x))=0$, and

$$
\begin{gathered}
\pi_{2}(P(x, y))=x^{2} y^{4}+y^{2}+1=\left(x y^{2}+y+1\right)^{2}, \\
\pi_{2}(Q(x, y))=x^{7} y^{8}+x^{3} y^{4}+x^{3}=x^{3}\left(x y^{2}+y+1\right)^{4},
\end{gathered}
$$

we have

$$
\begin{array}{lc}
x \varphi(x)^{2}+\varphi(x)+1 \equiv 0 & (\bmod 2), \\
x \psi(x)^{2}+\psi(x)+1 \equiv 0 & (\bmod 2),
\end{array}
$$

and therefore

$$
\begin{aligned}
& \left(x \varphi(x)^{2}+\varphi(x)+1\right)^{2} \equiv 0 \quad(\bmod 4), \\
& \left(x \psi(x)^{2}+\psi(x)+1\right)^{2} \equiv 0 \quad(\bmod 4) .
\end{aligned}
$$

In other words, the polynomial $S(x, y)=\left(x y^{2}+y+1\right)^{2} \in \mathbb{Z} / 4 \mathbb{Z}[x, y]$ is an annihilating polynomial for both $\bar{C}=\pi_{4}(\varphi)$ and $\bar{D}=\pi_{4}(\psi)$.

Now we prove that there is no polynomial in $\mathbb{Z} / 4 \mathbb{Z}[x, y]$ that, seen as a polynomial in $y$, has degree less than 4 , and, whose leading coefficient is invertible in the ring of Laurent series $\mathbb{Z} / 4 \mathbb{Z}((x))$, that annihilates either $\bar{C}(x)$ or $\bar{D}(x)$. By absurdity, suppose that $Q(x, y)=Q_{n}(x) y^{n}+\cdots+Q_{1}(x) y+Q_{0}(x)$ is such a polynomial of minimal degree on $y$. By assumption, $n$ is less than $4, Q_{n}(x)$ is invertible in $\mathbb{Z} / 4 \mathbb{Z}((x))$, and $Q(x, y)$ annihilates either $\bar{C}(x)$ or $\bar{D}(x)$. Since $Q_{n}(x)$ is invertible in $\mathbb{Z} / 4 \mathbb{Z}((x))$, we can effectuate Euclidean division of $P(x, y)$ by $Q(x, y)$, and by minimality of $n$, we obtain

$$
Q_{n}(x) P(x, y)=Q(x, y) R(x, y)
$$

for some $R(x, y) \in \mathbb{Z} / 4 \mathbb{Z}[x, y]$.

Reducing modulo 2 (where we use $\pi_{2}$ by abuse of notation), we get

$$
\pi_{2}(Q(x, y)) \pi_{2}(R(x, y))=\pi_{2}\left(Q_{n}(x) P(x, y)\right)=\pi_{2}\left(Q_{n}(x)\right)\left(x y^{2}+y+1\right)^{2} .
$$

Since $Q_{n}(x)$ is invertible in $\mathbb{Z} / 4 \mathbb{Z}((x))$, its projection $\pi_{2}(Q(x))$ is non-zero. As factorization into irreducible factors of $\pi_{2}(Q(x, y) R(x, y))$ in $\mathbb{F}_{2}(x)[y]$ is unique up to multiplication by elements in $\mathbb{F}_{2}(x)$, and $1 \leq n \leq 3$, we know that there exists $\alpha(x) \in \mathbb{Z}[x]$ taking coefficients in $\{0,1\}$, such that $\pi_{2}(\alpha(x))$ is a factor of $\pi_{2}\left(Q_{n}(x)\right)$ and

$$
\pi_{2}(Q(x, y))=\pi_{2}(\alpha(x)) \cdot\left(x y^{2}+y+1\right) .
$$

Therefore there exist polynomials $\beta_{0}(x), \beta_{1}(x), \beta_{2}(x)$ in $\mathbb{Z}[x]$ taking coefficients in $\{0,1\}$, such that

$$
Q(x, y)=\pi_{4}(\alpha(x)) \cdot\left(x y^{2}+y+1\right)+2 x \pi_{4}\left(\beta_{2}(x)\right) y^{2}+2 \pi_{4}\left(\beta_{1}(x)\right) y+2 \pi_{4}\left(\beta_{0}(x)\right) .
$$

Since, by assumption, $Q\left(x, \pi_{4}(f(x))\right)=0$, where $f$ stands for one of $C$ and $D$, we have

$$
\alpha(x)\left(x f(x)^{2}+f(x)+1\right) \equiv 2 x \beta_{2}(x) f(x)^{2}+2 \beta_{1}(x) f(x)+2 \beta_{0}(x) \quad(\bmod 4) .
$$

We let $g(x)$ denote the series $\left(x f(x)^{2}+f(x)+1\right) / 2$, by Eqs. (5.1) and (5.2) we know that $g(x)$ has integer coefficients. We rewrite the above congruence as

$$
\alpha(x) g(x) \equiv x \beta_{2}(x) f(x)^{2}+\beta_{1}(x) f(x)+\beta_{0}(x) \quad(\bmod 2),
$$

in other words,

$$
\pi_{2}(\alpha(x)) \pi_{2}(g(x))=x \pi_{2}(\beta(x)) \pi_{2}(f(x))^{2}+\pi_{2}\left(\beta_{1}(x)\right) \pi_{2}(f(x))+\pi_{2}\left(\beta_{0}(x)\right) .
$$


In light of Eqs. (5.1) and (5.2), $\pi_{2}(f(x))$ is of degree 2 over $\mathbb{F}_{2}(x)$, so that the right hand side of Eq. (5.3) lives in a quadratic extension of $\mathbb{F}_{2}(x)$. We will prove that the left hand side of Eq. (5.3) is of degree 4 over $\mathbb{F}_{2}(x)$, which will lead to a contradiction. Also, $\pi_{2}(\alpha(x))$ being a non-zero element in $\mathbb{F}_{2}(x)$, we only need to prove that the degree of $\pi_{2}(g(x))$ over $\mathbb{F}_{2}(x)$ is 4 .

In case $f(x)=C(x)$, since $C(x) \equiv \varphi(x)(\bmod 4)$, we have

$$
x C(x)^{2}+C(x)+1 \equiv x \varphi(x)^{2}+\varphi(x)+1 \quad(\bmod 4),
$$

and therefore

$$
g(x) \equiv\left(x \varphi(x)^{2}+\varphi(x)+1\right) / 2 \quad(\bmod 2) .
$$

From Theorem 1.1, we find that $\left(x \varphi(x)^{2}+\varphi(x)+1\right) / 2$ is equal to

$x \sqrt{-4 x+1}+x \sqrt{2 \sqrt{-4 x+1}-1}+\frac{1}{2} \sqrt{-4 x+1} \sqrt{2 \sqrt{-4 x+1}-1}+\frac{1}{2} \sqrt{-4 x+1}$.

Its annihilating polynomial is

$$
\begin{aligned}
T(x, y)= & 16 x^{6}+8 y^{2} x^{3}+32 y x^{4}-32 x^{5}+y^{4}+24 y^{2} x^{2}-40 y x^{3}+8 x^{4} \\
& -6 y^{2} x-8 y x^{2}+16 x^{3}+8 y x-8 x^{2}-y+x .
\end{aligned}
$$

Therefore $\pi_{2}(T(x, y))=y^{4}+y+x$ is an annihilating polynomial of $\pi_{2}(g(x))$. Let us verify that it is irreducible in $\mathbb{F}_{2}[x][y]$. If $y^{4}+y+x$ factorizes into a cubic and a linear factor, then the linear factor must be $(y+x)$ or $(y+1)$. However, $y^{4}+y+x$ is divisible by neither. If it factorizes into two quadratic factors, then it must be of the form $\left(y^{2}+\xi(x) y+1\right)\left(y^{2}+\eta(x) y+x\right)$, where $\xi(x)$ and $\eta(x)$ are in $\mathbb{F}_{2}[x]$. When we expand and compare the coefficients, we see that $\xi(x)$ and $\eta(x)$ must satisfy simultaneously $\xi(x)+\eta(x)=0$ and $\xi(x) \eta(x)+x+1=0$, which is impossible.

In case $f(x)=D(x)$, we find that

$$
g(x) \equiv\left(x \psi(x)^{2}+\psi(x)+1\right) / 2 \quad(\bmod 2) .
$$

We could have computed an annihilating polynomial for $\pi_{2}(g(x))$ the same way that we did in the case $f(x)=C(x)$, but we would have to deal with too many terms in the calculation involving $D(x)$. So we choose to work directly in $\mathbb{F}_{2}[[x]]$, by using Corollary 4.1 to find the 2-kernel of $\pi_{2}(g(x))$, from which we will obtain the minimal polynomial of $\pi_{2}(g(x))$ following the method in the proof of Theorem 1 from the work of Christol, Kamae, Mendès France and Rauzy [14].

We prove now that the structure of the 2-kernel of $\pi_{2}(g(x))$ is

$$
[[1,0],[2,3],[1,4],[3,4],[4,4]] \text {. }
$$

By Corollary 4.1,

$$
D(x) \equiv 1-x^{-1} S_{\infty}(x)^{2}+2 x^{-1} S_{\infty}^{o}(x)\left(1+S_{\infty}(x)\right) \quad(\bmod 4) .
$$

Therefore

$$
\begin{gathered}
D(x) \equiv 1+x^{-1} S_{\infty}(x)^{2} \equiv 1+\sum_{j=1}^{\infty} x^{2^{j}-1} \equiv \sum_{j=0}^{\infty} x^{2^{j}-1} \quad(\bmod 2) \\
x D(x)^{2} \equiv \sum_{i, j=0}^{\infty} x^{2^{i}+2^{j}-1} \quad(\bmod 4)
\end{gathered}
$$

and

$$
x D(x)^{2}+D(x)+1 \equiv 2+2 \sum_{k=0}^{\infty} x^{2^{2 k+1}-1}\left(1+\sum_{j=0}^{\infty} x^{2^{j}}\right) \quad(\bmod 4)
$$


We now have an explicit expression for $\pi_{2}(g(x))$

$$
\pi_{2}(g(x))=1+1 \sum_{k=0}^{\infty} x^{2^{2 k+1}-1}\left(1+\sum_{j=0}^{\infty} x^{2^{j}}\right)=: g_{0}(x) .
$$

To compute the 2-kernel of $g_{0}(x)=\pi_{2}(g(x))$, we apply the operators $\Lambda_{0}$ and $\Lambda_{1}$ :

$$
\begin{gathered}
\Lambda_{0} g_{0}(x)=1+\sum_{k=0}^{\infty} x^{2^{2 k}}=: g_{1}(x), \\
\Lambda_{1} g_{0}(x)=g_{0}(x), \\
\Lambda_{0}\left(g_{1}(x)\right)=1+\sum_{k=0}^{\infty} x^{2^{2 k+1}}=: g_{2}(x), \\
\Lambda_{1} g_{1}(x)=1=: g_{3}(x), \\
\Lambda_{0} g_{2}(x)=g_{1}(x), \\
\Lambda_{1} g_{2}(x)=0=: g_{4}(x) .
\end{gathered}
$$

Therefore the 2-kernel of $g$ is

$$
[[1,0],[2,3],[1,4],[3,4],[4,4]] .
$$

The following identities are just another way of writing out the 2-kernel.

$$
\begin{gathered}
g_{0}(x)=g_{1}(x)^{2}+x g_{0}(x)^{2}, \\
g_{1}(x)=g_{2}(x)^{2}+x g_{3}(x)^{2}=g_{2}(x)^{2}+x, \\
g_{2}(x)=g_{1}(x)^{2}+x g_{4}(x)^{2}=g_{1}(x)^{2} .
\end{gathered}
$$

From these, we deduce that $\pi_{2}(g(x))=g_{0}(x)$ is a root of the polynomial

$$
x^{4} y^{8}+y^{4}+x y^{2}+y+x^{2}
$$

in $\mathbb{F}_{2}[x, y]$, which factorizes as

$$
\left(x y^{4}+y^{3}+1\right)\left(x^{3} y^{4}+x^{2} y^{3}+x y^{2}+y+x^{2}\right) .
$$

By computing the first few terms of $\pi_{2}(g(x))$ we find that the second factor is not an annihilating polynomial for $\pi_{2}(g(x))$, and therefore $x y^{4}+y^{3}+1$ is. As

$$
x y^{4}+y^{3}+1=y^{4}\left(\left(\frac{1}{y}\right)^{4}+\frac{1}{y}+x\right),
$$

and we have just shown in the case $f=C(x)$ that $y^{4}+y+x$ is irreducible in $\mathbb{F}_{2}[x, y], x y^{4}+y^{3}+1$ is also irreducible. This shows that $\pi_{2}(g(x))$ has degree 4 over $\mathbb{F}_{2}(x)$ and completes our proof. 


\section{HANKEL DETERMinANTS}

The Hankel determinants of $C(x)$ and $D(x)$ can be calculated by Heilermann's theorem. To prove their automacity, we need the following theorem.

Theorem 6.1 (see [2]). Let $X$ be an alphabet on which is defined an associative operation $*$. Let $x=\left(x_{n}\right)$ be a q-automatic sequence on the alphabet $X$. The sequence $y=\left(y_{n}\right)$ defined by

$$
\begin{aligned}
y_{1} & =x_{0} \\
y_{2} & =x_{1} * x_{0} \\
& \vdots \\
y_{n} & =x_{n-1} * x_{n-2} * \cdots * x_{0}
\end{aligned}
$$

is q-automatic.

From the definition of the Thue-Morse and the period-doubling sequence, it is easy to see that

$$
t_{2 k+1} t_{2 k+2}=s_{k}, \quad \text { and } \quad s_{2 k+1} s_{2 k+2}=-s_{k}
$$

By Theorem 2.2, we have

$$
\begin{aligned}
H_{n}(C(x)) & =t_{0}^{n}\left(t_{1} t_{2}\right)^{n-1}\left(t_{3} t_{4}\right)^{n-2} \cdots\left(t_{2 n-3} t_{2 n-2}\right)^{1} \\
& =s_{0}^{n-1} s_{1}^{n-2} \cdots s_{n-2}^{1} .
\end{aligned}
$$

and

$$
\begin{aligned}
H_{n}(D(x)) & =s_{0}^{n}\left(s_{1} s_{2}\right)^{n-1}\left(s_{3} s_{4}\right)^{n-2} \cdots\left(s_{2 n-3} s_{2 n-2}\right)^{1} \\
& =(-1)^{n(n-1) / 2} s_{0}^{n-1} s_{1}^{n-2} \cdots s_{n-2}^{1} \\
& =(-1)^{n(n-1) / 2} H_{n}(C(x)) .
\end{aligned}
$$

Define $u_{n}:=s_{0} s_{1} \cdots s_{n-1}$. By Theorem 6.1, $\left(u_{n}\right)$ is 2-automatic, and consequently $H_{n}(C(x))=u_{0} u_{1} \cdots u_{n-1}$ is 2-automatic. Since $(-1)^{n(n-1) / 2}$ is periodic, $H_{n}(D(x))$ is also 2-automatic. Finally $H_{n}(\bar{C}(x))$ and $H_{n}(\bar{C}(x))$ are 2-automatic as the reduction modulo 4 of $H_{n}(C(x))$ and $H_{n}(D(x))$.

Acknowledgments The authors would like to thank Jean-Paul Allouche for valuable suggestions and Zhi-Ying Wen for invitation to Tsinghua University which facilitated collaboration.

\section{REFERENCES}

[1] Jean-Paul Allouche. Sur le développement en fraction continue de certaines séries formelles. C. R. Acad. Sci. Paris Sér. I Math., 307(12):631-633, 1988.

[2] Jean-Paul Allouche and Michel Mendès France. Quasicrystal Ising chain and automata theory. J. Statist. Phys., 42(5-6):809-821, 1986.

[3] Jean-Paul Allouche, Jacques Peyrière, Zhi-Xiong Wen, and Zhi-Ying Wen. Hankel determinants of the Thue-Morse sequence. Ann. Inst. Fourier (Grenoble), 48(1):1-27, 1998.

[4] Jean-Paul Allouche and Jeffrey Shallit. The ubiquitous Prouhet-Thue-Morse sequence. In Sequences and their applications (Singapore, 1998), Springer Ser. Discrete Math. Theor. Comput. Sci., pages 1-16. Springer, London, 1999.

[5] Jean-Paul Allouche and Jeffrey Shallit. Automatic sequences. Cambridge University Press, Cambridge, 2003. Theory, applications, generalizations.

[6] Dmitry Badziahin. Continued fractions of certain Mahler functions. Acta Arith., 188(1):5381, 2019. 
[7] Leonard E. Baum and Melvin M. Sweet. Continued fractions of algebraic power series in characteristic 2. Ann. of Math. (2), 103(3):593-610, 1976.

[8] Leonard E. Baum and Melvin M. Sweet. Badly approximable power series in characteristic 2. Ann. of Math. (2), 105(3):573-580, 1977.

[9] Petter Brändén, Anders Claesson, and Einar Steingrímsson. Catalan continued fractions and increasing subsequences in permutations. Discrete Math., 258(1-3):275-287, 2002.

[10] Yann Bugeaud. On the rational approximation to the Thue-Morse-Mahler numbers. Ann. Inst. Fourier (Grenoble), 61(5):2065-2076 (2012), 2011.

[11] Yann Bugeaud. Automatic continued fractions are transcendental or quadratic. Ann. Sci. Éc. Norm. Supér. (4), 46(6):1005-1022, 2013.

[12] Yann Bugeaud and Guo-Niu Han. A combinatorial proof of the non-vanishing of Hankel determinants of the Thue-Morse sequence. Electron. J. Combin., 21(3):Paper 3.26, 17, 2014.

[13] Yann Bugeaud, Guo-Niu Han, Zhi-Ying Wen, and Jia-Yan Yao. Hankel determinants, Padé approximations, and irrationality exponents. Int. Math. Res. Not. IMRN, (5):1467-1496, 2016.

[14] Gilles Christol, Teturo Kamae, Michel Mendès France, and Gérard Rauzy. Suites algébriques, automates et substitutions. Bull. Soc. Math. France, 108(4):401-419, 1980.

[15] Michael Coons and Paul Vrbik. An irrationality measure for regular paperfolding numbers. J. Integer Seq., 15(1):Article 12.1.6, 10, 2012.

[16] Jan Denef and Leonard Lipshitz. Algebraic power series and diagonals. J. Number Theory, 26(1):46-67, 1987.

[17] Philippe Flajolet. Combinatorial aspects of continued fractions. Discrete Math., 32(2):125$161,1980$.

[18] Dominique Foata and Guo-Niu Han. Principes de combinatoire classique. Lecture notes, Strasbourg, 2000.

[19] Robbert J. Fokkink, Cor Kraaikamp, and Jeffrey Shallit. Hankel matrices for the perioddoubling sequence. Indag. Math. (N.S.), 28(1):108-119, 2017.

[20] Hao Fu and Guo-Niu Han. Computer assisted proof for Apwenian sequences. In Proceedings of the 2016 ACM International Symposium on Symbolic and Algebraic Computation, pages 231-238. ACM, New York, 2016.

[21] Ying-Jun Guo, Zhi-Xiong Wen, and Wen Wu. On the irrationality exponent of the regular paperfolding numbers. Linear Algebra Appl., 446:237-264, 2014.

[22] Guo-Niu Han. Hankel determinant calculus for the Thue-Morse and related sequences. J. Number Theory, 147:374-395, 2015.

[23] Guo-Niu Han. Hankel continued fraction and its applications. Adv. Math., 303:295-321, 2016.

[24] J. B. H. Heilermann. Über die Verwandlung der Reihen in Kettenbrüche. J. Reine Angew. Math., 33:174-188, 1846.

[25] William B. Jones and Wolfgang J. Thron. Continued fractions - Analytic theory and applications, volume 11 of Encyclopedia of Mathematics and its Applications. Addison-Wesley Publishing Co., Reading, Mass., 1980.

[26] Alain Lasjaunias and Jia-Yan Yao. Hyperquadratic continued fractions in odd characteristic with partial quotients of degree one. J. Number Theory, 149:259-284, 2015.

[27] Alain Lasjaunias and Jia-Yan Yao. Hyperquadratic continued fractions and automatic sequences. Finite Fields Appl., 40:46-60, 2016.

[28] Alain Lasjaunias and Jia-Yan Yao. On certain recurrent and automatic sequences in finite fields. J. Algebra, 478:133-152, 2017.

[29] W. H. Mills and David P. Robbins. Continued fractions for certain algebraic power series. $J$. Number Theory, 23(3):388-404, 1986.

[30] Oskar Perron. Die Lehre von den Kettenbrüchen. Dritte, verbesserte und erweiterte Aufl. Bd. II. Analytisch-funktionentheoretische Kettenbrüche. B. G. Teubner Verlagsgesellschaft, Stuttgart, 1957.

[31] Luke Schaeffer and Jeffrey Shallit. Closed, palindromic, rich, privileged, trapezoidal, and balanced words in automatic sequences. Electron. J. Combin., 23(1):Paper 1.25, 19, 2016.

[32] Thomas Jan Stieltjes. Recherches sur les fractions continues. Ann. Fac. Sci. Toulouse Math. (6), 4(4):A5-A47, 1995. Reprint of Ann. Fac. Sci. Toulouse 9 (1895), A5-A47.

[33] Axel Thue. Über die gegenseitige Lage gleicher Teile gewisser Zeichenreihen. Kra. Vidensk. Selsk. Skrifer, I. Mat. Nat. Kl., pages 1-67, 1912. 
[34] Gérard Viennot. Une théorie combinatoire des polynômes orthogonaux généraux. Departement de Mathématiques et d'Informatique, Université du Québec à Montréal, 1983.

[35] Hubert S. Wall. Analytic Theory of Continued Fractions. D. Van Nostrand Company, Inc., New York, N. Y., 1948.

School of Mathematics and Statistics, Huazhong University of Science and TechNOLOGY, WuHAN, PR ChinA

Email address: huyining@hust.edu.cn

Université de Strasbourg, CNRS, IRMA UMR 7501, F-67000 Strasbourg, France

Email address: guoniu.han@unistra.fr 